\title{
Methamphetamine promotes a-tubulin deacetylation in endothelial cells: The protective role of acetyl-L-carnitine
}

S. Fernandes a, b, c, d, S. Salta a, b , c, T. Summavielle *,a , b, c a

a) Rua Alfredo Allen, Instituto de Investigação e Inovação em Saúde, Universidade do Porto, 4200-135 Porto, Portugal

b) Rua do Campo Alegre, 823, Addiction Biology Group, Instituto de Biologia Molecular e Celular (IBMC), Universidade do Porto, 4150-180 Porto, Portugal

c) Rua Valente Perfeito, 322, School of Allied Health Sciences - Polytechnic Institute of Porto (ESTSP-IPP), 4400-330 Vila Nova de Gaia, Portugal

d) Alameda Prof. Hernâni Monteiro, Faculdade de Medicina da Universidade do Porto (FMUP), 4200-319 Porto, Portugal

\begin{abstract}
Methamphetamine (METH) is a powerful psychostimulant drug used worldwide for its reinforcing properties. In addition to the classic long-lasting monoaminergic-disrupting effects extensively described in the literature, METH has been consistently reported to increase blood brain barrier (BBB) permeability, both in vivo and in vitro, as a result of tight junction and cytoskeleton disarrangement. Microtubules play a critical role in cell stability, which relies on post-translational modifications such as a-tubulin acetylation. As there is evidence that psychostimulants drugs modulate the expression of histone deacetylases (HDACs), we hypothesized that in endothelial cells METH-mediation of cytoplasmatic HDAC6 activity could affect tubulin acetylation and further contribute to BBB dysfunction. To validate our hypothesis, we exposed the bEnd. 3 endothelial cells to increasing doses of METH and verified that itleads to an extensivea-tubulin deacetylation mediated by HDACs activation. Furthermore, since we recently reported that acetyl-L-carnitine (ALC), a natural occurring compound, prevents BBB structural loss in a context of METH exposure, we reasoned that ALC could also preserve the acetylation of microtubules under METH action. The present results confirm that ALC is able to prevent METH-induced deacetylation providing effective protection on microtubule acetylation. Although further investigation is still needed, HDACs regulation may become a new therapeutic target for ALC.
\end{abstract}

Keywords: Cytoskeleton; a-Tubulin; Methamphetamine; Acetyl-L-carnitine; Histone deacetylase

1. Introduction Methamphetamine (METH) is a powerful psychostimulant used worldwide for its reinforcing properties that leads to long-lasting deleterious effects (Gold et al., 2009; Krasnova and Cadet, 2009).

METH toxicity is characterized by the disruption of the dopaminergic system, concomitant with terminal degeneration and eventual neuronal death (Conant et al., 2011; Krasnova and Cadet, 2009). However, METH has been increasingly recognized to impact also the blood-brain-barrier (BBB), causing the release of inflammatory mediators and astrogliosis (Gold et al., 2009; Goncalves et al., 2010; Northrop and 
Yamamoto, 2012; Ramirez et al., 2009). METH-induced permeability at the BBB level has been consistently reported both in vivo and in vitro (Conant et al., 2011; Martins et al., 2011; Urrutia et al., 2013), as a result of tight junction and cytoskeleton disarrangement (Dietrich, 2009; Kousik et al., 2012; Park et al., 2013). In endothelial cells, METH was also shown to trigger nitric oxide (NO)-mediated transcytosis (Martins et al., 2013). Recently, we showed, also in endothelial cells, that exposure to METH leads to disruption of actin filaments concomitant with claudin-5 translocation to the cytoplasm, promoted by MMP-9 activation in association with ILK overexpression (Fernandes et al., 2014). Similarly to the actin filaments, microtubules play a critical role in cell stability and dynamics. Proper regulation of microtubule components relies on post translational modifications such as a-tubulin acetylation (Hammond et al., 2008). Microtubule deacetylation is carried out by histone deacetylase (HDAC) 6, a class II HDAC and the class III HDAC sirtuin 2 (SIRT2), which form a complex that allows them to bind to tubulin (Hubbert et al., 2002; Nahhas et al., 2007; Sadoul et al., 2011; Yang and Seto, 2008). Of note, it was recently shown that interfering with HDAC6 is sufficient to prevent microtubule deacetylation (Gold et al., 2015). There is growing evidence that HDACs inhibition is strongly associated with decreased cell mobility, which is of particular interest in the oncology field (Hrabeta et al., 2014; Ocker and Schneider-Stock, 2007). Although there are several studies showing that METH and other psychostimulants affect the expression of HDACs (Cassel et al., 2006; Host et al., 2011; Martin et al., 2012; Omonijo et al., 2014), and in particular the expression of HDAC6 (Omonijo et al., 2014), the effect of METH in microtubules acetylation was not yet explored. Therefore, we hypothesized that METH-induced regulation of HDACs activity, and in particular of HDAC6, may also mediate the structural loss observed in METH-exposed endothelial cells. Moreover, since we recently showed that a pretreatment with acetyl-L-carnitine (ALC) was able to prevent METH-induced activation of MMP-9, preserving the actin structural arrangement in the endothelial cells (Fernandes et al., 2014), and ALC was shown to have the potential to interact with HDAC activity (Huang et al., 2012), we reasoned that ALC could also preserve the acetylation of microtubules under METH action. ALC is a natural occurring compound that was seen to be protective by different mechanisms in several neurological conditions, including BBB dysfunction (Alves et al., 2009; Haorah et al., 2011; Muneer et al., 2011; Pettegrew et al., 2000). To verify our hypothesis, we exposed the endothelial cell line bEnd. 3 to increasing doses of METH and evaluated the individual and combined action of METH and ALC on a-tubulin acetylation. Trichostatin A (TSA), a natural product isolated from Streptomyces hygroscopicus commonly used as an inhibitor of class I/II HDACs known to promote microtubule acetylation, was also assayed (Dompierre et al., 2007; Harrison and Dexter, 2013).

\section{Material and methods}

\subsection{In vitro model and cell culture}

The immortalized bEnd. 3 cells are derived from mouse brains and known to mimic some of the BBB characteristics. The cell line bEnd. 3 was obtained from ATCC (American Type Cell Culture-CRL- 2299, Manassas, VA) and cultures were maintained in DMEM (1)/ Glutamax (GIBCO1, Life Technologies, Paisley, UK), containing $1 \%$ penicillin and streptomycin (GIBCO1, Life Technologies) and 10\% fetal bovine serum (GIBCO1, Life Technologies). Purity of the cell line was checked using an anti-CD31 antibody (Abcam 7388 , rat monoclonal, 1:1000), which showed $100 \%$ enrichment of cells on the adhesion 
marker. For immunocytochemistry, cells were plated on 24-well plates $(80,000$ cells/well) containing glass cover slips. To obtain protein extracts or mRNA, bEnd. 3 cells were cultured in petri dishes ( 1 million of cells). Cell culture media was changed every 3 days until cells were confluent.

\subsection{Drug regimen}

ALC-hydrochloride was kindly provided by Sigma-Tau S.p.A (Pomezia, Italy). TSA was purchased from Promega (\#G6560) and METH hydrochloride from Sigma-Aldrich (St. Louis, MO, Cat. M-8750). Immortalized bEnd. 3 cells at confluence were treated with 0.5 $\mathrm{mM}$ and $1 \mathrm{mM}$ of METH. METH doses were predetermined in our laboratory in agreement with previous studies using METH in similar cell models (Fernandes et al., 2014; Jin et al., 2002). ALC $1 \mathrm{mM}$ was added $30 \mathrm{~min}$ before METH. TSA was used in a 100 $\mathrm{nM}$ concentration. The selected TSA and ALC doses were previously shown to be safe for the cells and are below the range of doses previously used in similar works (Huang et al., 2012; Hubbert et al., 2002; Tu et al., 2014).

\subsection{Immunostaining procedure}

For immunocytochemistry, bEnd. 3 cells were cultured on glass cover slips in 24 well plates until $90-100 \%$ confluence. Cells were then treated with 0.5 and $1 \mathrm{mM}$ of METH in the presence or absence of $100 \mathrm{nM}$ TSA or $1 \mathrm{mM} \mathrm{ALC}$, for $24 \mathrm{~h}$. To evaluate a-tubulin, cells were washed with PBS, fixed during $10 \mathrm{~min}$ in methanol and permeabilized in $0.1 \%$ Triton X-100 during 10 min. After a blocking of $45 \mathrm{~min}$ in 10\% NGS (normal goat serum), cells were incubated overnight at $4 \mathrm{C}$ with respective primary antibodies - Mouse antia-tubulin (Sigma, clone AA13 IgG1, \#T8203, 1:500) and Mouse anti-acetyl-a-tubulin (Sigma, clone 6-11B-1 IgG2b, \#T7451, 1:500). For secondary antibody incubation we used Anti-Mouse IgG Alexa-Fluor 4881 Conjugate (Life Technologies, \#A11001, 1:1000) for acetylated form and Anti-Mouse IgG Alexa-Fluor 5681 Conjugate (Life Technologies, \#A11004, 1:1000) for total a-tubulin, for $1 \mathrm{~h}$ at room temperature in the dark. Coverslips were then mounted onto glass slides with immunomount (Fluorescent Mounting Medium, CA, USA) containing 40 ,6-diamidino-2-phenylindole (DAPI), and then fluorescence microphotographs were captured using Axio Imager Z1 fluorescence microscope (Carl Zeiss, Germany).

\subsection{Morphometric analysis}

Changes observed in a-tubulin acetylation by immunofluorescence assays were evaluated using the Fiji Software version 2.0. A total of four independent experiments were performed. From each coverslip, ten images were blindly captured and analyzed through measurement of fluorescence intensity.

\subsection{Protein expression analysis by western blot}

Confluent bEnd. 3 cells cultured in petri dishes were scrapped and lysed with TEN buffer (50 mM Tris- $\mathrm{HCl}, 2 \mathrm{mM}$ EDTA, $150 \mathrm{mM} \mathrm{NaCl}, 1 \% \mathrm{NP}-40$, supplemented with phosphatases and proteases inhibitors) and then centrifuged at 14,000 $\mathrm{g}$ for $15 \mathrm{~min}$ at 4 ㄷ. Protein concentration of the cell lysate was estimated in the supernatant by the Bradford method (Bio-Rad Protein Assay, Munich, Germany). Proteins were loaded at $25 \mathrm{mg}$ per lane and resolved by SDS-PAGE on $12 \%$ Bis/acrilamide gels and then transferred onto PVDF membranes. After blocking with dry milk 5\%, membranes were 
incubated with mouse anti-acetyl-a-tubulin antibody (Sigma, clone 6-11B-1 IgG2b, \#T7451,1:1600). Membranes were washed in Stripping Buffer (RestoreTM Western Blot, Thermo Scientific) and then incubated with mouse anti-a-tubulin (Sigma, clone AA13 IgG1, \#T8203, 1:1000). Secondary antibody HRP (horseradish peroxidase)-conjugated were used for $1 \mathrm{~h}$ of incubation. Chemiluminescent signal detection was achieved using the Immun-Star HRP kit (Bio-Rad Laboratories, USA). A GS800 densitometer (Bio-Rad) and quantity one 1-D analysis software (v4.6, Bio-Rad) were used for densitometry analysis.

\subsection{Measurement of HDACs I/II activity}

Evaluation of HDACs activity was performed using HDAC-GloTM I/II Assay and Screening System kit (Promega, USA) following provided instructions. This luminescent assay measures relative activity of HDAC enzymes using an acetylated luminogenic peptide susbstrate. When cleaved the substrate is quantified in a reaction using Ultra-GloTM Recombinant Luciferase. Briefly, in each well of 96 multiwell plates, 10,000 bEnd. 3 cells were seeded and treated with ALC and TSA in dilution series: ALC (from $2 \mathrm{mM}$ to 0.125 $\mathrm{mM}$ ), and TSA (from $500 \mathrm{nM}$ to $3.9 \mathrm{nM}$ ). $24 \mathrm{~h}$ after treatment, medium was replaced by another without phenol red and HDAC buffer was added, followed by HDACTM I/II reagent. After an incubation of $30 \mathrm{~min}$, luminescence was measured (Gen5 Data Analysis Software, Biotek Instruments, Inc., Synergy 2 (Izasa).

\subsection{Real time $-P C R$}

Total RNA was extracted from confluent bEnd.3 cells grown in petri dishes, using RNeasy1 Mini Kit (Qiagen, Hilden, Germany). RNA quality was checked by Experion automated electrophoresis system (Bio-Rad Hercules, CA, USA) and CDNA synthesis was performed by Qiagen RT2 HT First Strand cDNA kit using 2.0mg of RNA. RT2 SYBR Green Mastermix was used to amplify and quantify genes expression on a cycler (Bio-Rad iQ5 model). Specific primers were designed for gene relative expression of CDKN1A ( $F: 50$ TGACAGATTTCTATCACTCCAAG30; R: 50 TGACCCACAGCAGAAGAG30) and HDAC6 (F: 50 GCAGGAGGCAAGTTGATT30 ; R: 50 AAGAAGGGTGTGGAGTGA30). Data were analyzed by DDCT method after normalization to GUSB (F: 50 GGTGAAGGTGACAACAACT30 ; R: 50 CTGAATCCTCGTGCTTATTGA30 ) housekeeping gene expression.

\subsection{Statistical analysis}

Results from at least four independent experiments were represented as mean SEM. Significant differences between groups were determined by one-way ANOVA followed by the Sidak's post hoc test. Significance was set at $p<0.05$. All analysis were conducted using the software GraphPad Prism1 6.0 for Mac OSX (GraphPad Software, La Jolla, CA).

3. Results

\subsection{METH decreased a-tubulin acetylation}

The present results show that METH decreased a-tubulin acetylation. Immunofluorescence assays were done for both total and acetylated forms of a-tubulin showing that when bEnd. 3 cells were exposed to METH $1 \mathrm{mM}$, the amount of the acetylated form was significantly reduced ( $p<0.01$, Fig. 1a and $c$ ). No significant 
differences were seen in total a-tubulin (Fig. 1b). This was confirmed by western blot analysis where METH $1 \mathrm{mM}$ reduced significantly the rate of acetylated a-tubulin after $24 \mathrm{~h}$ of treatment ( $p<0.001$, Fig. $1 d)$.

a
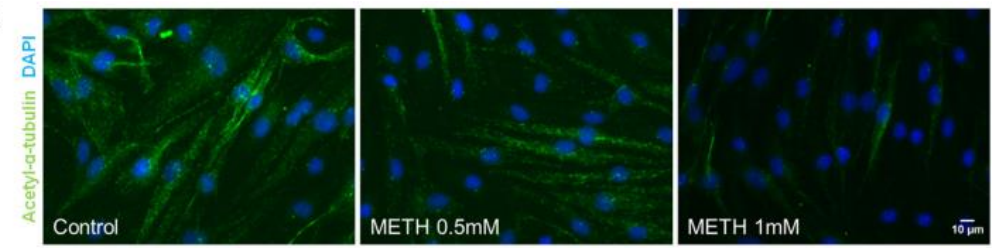

b
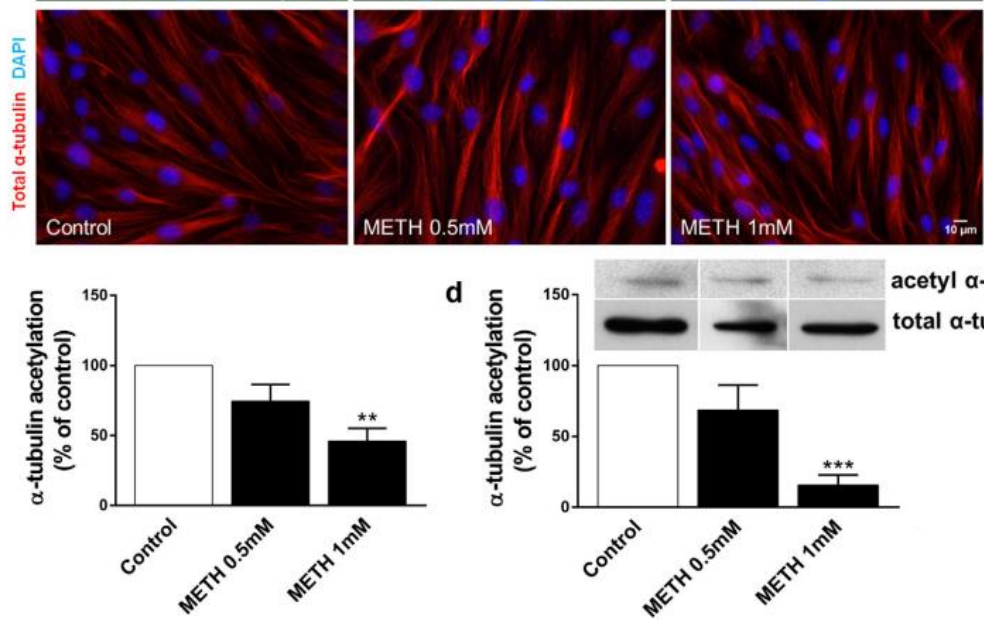

acetyl $\alpha$-tubulin total a-tubulin

Fig. 1. Quantification of $\alpha$-tubulin acetylation after METH exposure. Confluent bEnd.3 cells monolayers were treated with METH $0.5 \mathrm{mM}$ and $1 \mathrm{mM}$ doses according to the described procedures. Cells were stained for acetylated $\alpha$-tubulin (a) and total $\alpha$-tubulin (b). Nuclei were labeled with DAPI. Changes on acetylation, quantified through immunofluorescence intensity using the Fiji Software are shown in (c). Western blot was performed to assess the rate of acetylated $\alpha$-tubulin, the results are represented in (d). A minimum of five independent experiments were performed. Results were expressed as mean \pm SEM. ${ }^{* *} p<0.01$ and ${ }^{* * *} p<0.001$ compared to the control group (as determined by ANOVA followed by the Sidak's post-hoc test)

\subsection{ALC effectively prevented METH-induced a-tubulin deacetylation}

In the present study, we addressed the effect of ALC in preventing METH-induced deacetylation. Since HDAC6 is responsible for the a-tubulin deacetylation, we used a preselected dose of TSA (see Fig. 3a), a strong inhibitor of HDACs activity. Both ALC and TSA were able to counteract METH-induced deacetylation, as shown in Fig. 2a and b. Our results show that cytoskeleton tubulin from cells pretreated with $1 \mathrm{mM} \mathrm{ALC}$ is partially protected from METH-induced damage (Fig. 2a and b, $p<0.05$, METH $1 \mathrm{mM}$ vs. ALC/METH $1 \mathrm{mM}$ ). As expected, cells treated simultaneously with TSA and METH presented significantly increased acetylation as shown in Fig. $2 b(p<0.001$, TSA/METH $1 \mathrm{mM}$ vs. METH $1 \mathrm{~m}$ or vs. the control group). TSA by itself also led to a strong increase in a-tubulin acetylation ( $p<0.001$, TSA vs. control $100 \mathrm{nM}$ ). Further testing through western blot analysis confirmed these results. TSA $100 \mathrm{nM}$ alone or in combination with $M E T H$, resulted in a strong acetylation of a-tubulin compared to the control cells or to METH-exposed cells (Fig. 2a-c, $p<0.001$ for control vs. TSA $100 \mathrm{nM}$, control vs. TSA/ METH $1 \mathrm{mM}$, and METH $1 \mathrm{mM}$ vs. TSA/METH $1 \mathrm{mM}$ ). Pretreatment with ALC $1 \mathrm{mM}$ was also seen to be effective in preventing METHinduced deacetylation (Fig. 2c, $p<0.05$, METH $1 \mathrm{mM}$ vs. ALC/METH $1 \mathrm{mM}$ ). ALC by itself did not significantly differ from the control (Fig. $2 b$ and $c, p=0.42$ ). 

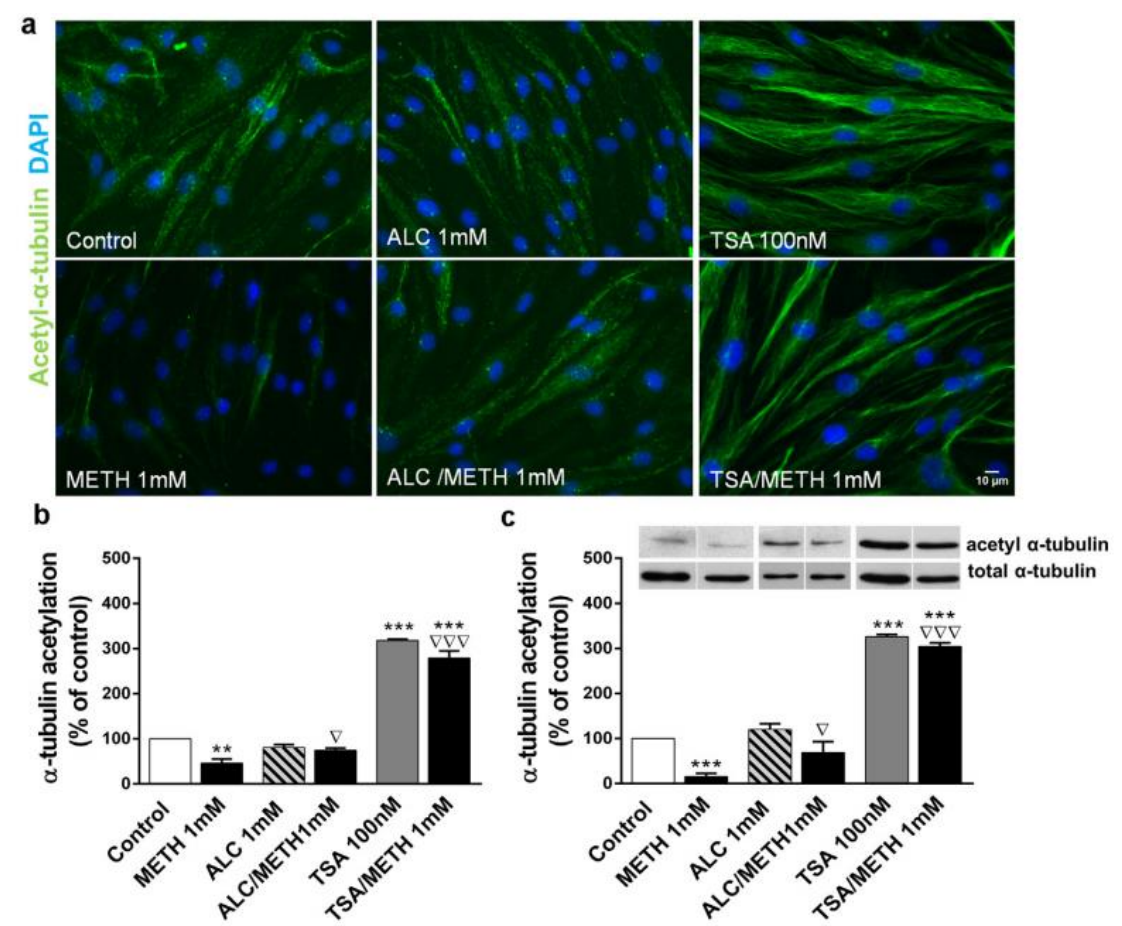

Fig. 2. Quantification of $\alpha$-tubulin acetylation in cells pretreated with ALC. Confluent bEnd.3 cells monolayers were treated with METH $1 \mathrm{mM}$ for $24 \mathrm{~h}$, with or without a pretreatment of $1 \mathrm{mM}$ ALC. TSA in a dose of $100 \mathrm{nM}$, alone or in combination with $1 \mathrm{mM}$ METH was used as positive control. Cells were stained for acetylated $\alpha$-tubulin and the nuclei were labeled with DAPI (a). Changes on acetylation were quantified through immunofluorescence intensity using the Fiji Software, and are represented in (b). Western blot was performed to assess the percentage of $\alpha$-tubulin acetylation in each condition. The obtained results are represented in (c). A minimum of five independent experiments was performed. Results were expressed as the mean \pm SEM. ${ }^{*} p<0.01$ and $\cdots p<0.001$ compared to control group; $\nabla p<0.05$ and $\nabla \nabla \nabla p<0.001$ compared to METH-treated cells (as determined by ANOVA followed by Sidak's post-hoc test).

\subsection{ALC is not an effective HDACs inhibitor}

Next, we investigated if ALC could act as a HDACs inhibitor. The effect of TSA, a strong inhibitor of HDACs was also assessed. As represented in Fig. 3a, a dose of $100 \mathrm{nM}$ TSA inhibits half of the HDACs activity, while a dose of $500 \mathrm{nM}$ reduced activity to near $20 \%$. ALC, however does not seem to be able to prevent HDACs activity (Fig. 3b), the $1 \mathrm{mM}$ dose seems to provide a slight reduction of HDACs activity, but fails to reach significance ( $p=0.513$, ALC $1 \mathrm{mM}$ vs. control).
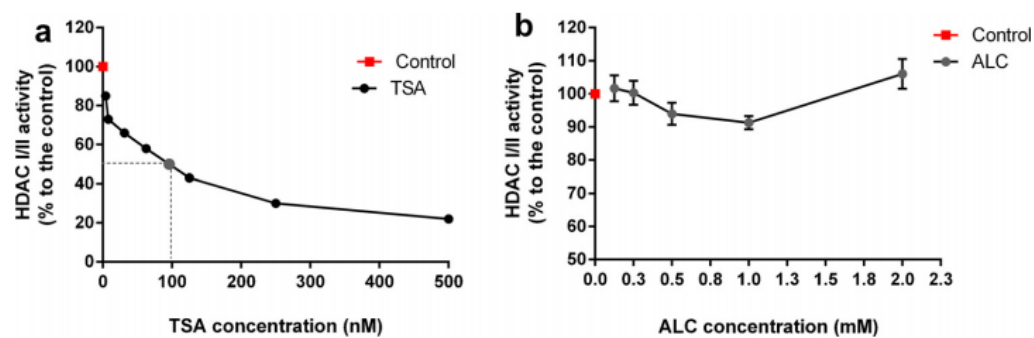

Fig. 3. Quantification of HDACs I/II class activity. Confluent bEnd.3 cells monolayers were treated with TSA in a dose ranging from from $3.9 \mathrm{nM}$ to $500 \mathrm{nM}$ (a) and ALC in doses ranging from $0.125 \mathrm{mM}$ to $2 \mathrm{mM}$ (b). HDACs I/II activity was assessed using HDAC-Glo ${ }^{\mathrm{TM}} \mathrm{I} / \mathrm{II}$ Assay and Screening System kit (Promega, USA) following provided instructions. Samples of ALC treated cells were evaluated in triplicate and results were measured through luminescence signal and are expressed as mean \pm SEM. TSA was used as a wellknown inhibitor of class I/II HDACs.

\subsection{ALC prevents METH-induced decrease in CDKN1A gene expression}

As ALC does not seem to act as a HDACs inhibitor, we investigated if it could act by downregulation of HDAC6 expression levels. However, as represented in Fig. 4a, the expression of the HDAC6 gene was not affected by METH or ALC, which seems to indicate that METH modulation of HDAC6 occurs at a post-translational level. A common marker of HDACs activity is the inhibition of CDKN1A gene expression due to chromatin 
remodeling and condensation (Xu et al., 2007). To evaluate the effect of METH and ALC over HDACs activity, we used RT-PCR to assess CDKN1A gene expression levels. As expected based on the effects described in Sections 3.1 and 3.2, METH-exposure induced a down-regulation of the CDKN1A gene (shown in Fig. $4 b, p<0.001$, control vs. METH $1 \mathrm{mM}$ ). TSA on the contrary, led to an up-regulation of the CDKN1A gene (Fig. 4b, $\mathrm{p}<0.05$, control vs. TSA $100 \mathrm{nM}$ ) and prevents the down-regulation induced by METH (Fig. 4b, $\mathrm{p}<0.001 \mathrm{METH} 1 \mathrm{mM}$ vs. TSA/METH $1 \mathrm{mM}$ ). Importantly, although ALC by itself did not affect the expression of the CDKN1A gene, a pretreatment with ALC was effective in preventing METH-induced CDKN1A down-regulation (Fig. 4b, $p<0.001$, METH vs. ALC/METH 1 mM).
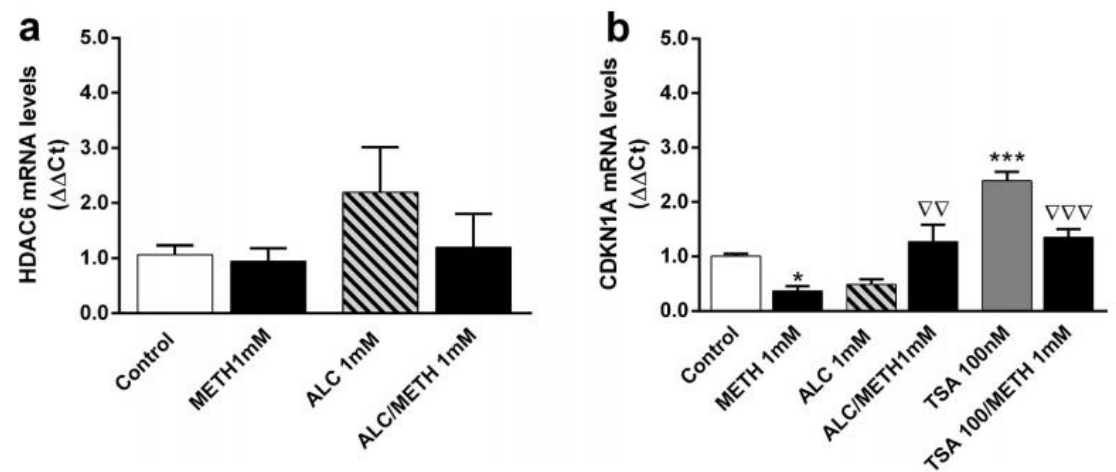

Fig. 4. Assessment of mRNA transcription. Confluent bEnd.3 cells monolayers were treated according to the described procedures. RNA was extracted from cultures treated with METH $1 \mathrm{mM}$, ALC $1 \mathrm{mM}$, TSA $100 \mathrm{nM}$ or combinations of such treatments, control cultures did not receive any treatment. For each PCR a total of $2.0 \mu \mathrm{g}$ RNA was used to determine gene expression of both HDAC6 (a) and CDKN1A (b). A total of four independent experiments were performed. Data normalization was made using GUSB gene expression. Results are expressed as mean \pm SEM. ${ }^{*} p<0.05$ and ${ }^{\cdots *} p<0.001$ compared to control group; $\nabla \nabla \nabla p<0.001$ compared to METH-treated cells (as determined by ANOVA followed by Sidak's post-hoc test).

4. Discussion

The present work shows that METH considerably reduces a-tubulin acetylation in endothelial cells, which is likely to impact cytoskeleton stability, cell motility and polarity, and vesicle transport (Hubbert et al., 2002; Matsuyama et al., 2002). Importantly, we show that ALC partially prevents METH-induced deacetylation, protecting the endothelial cells and consequently preserving the BBB function. Cytoskeleton microtubules are regulated by several types of conserved posttranslational modifications (Song and Brady, 2014). Although the biological meaning of such mechanisms is still poorly understood, it was reported that tubulin acetylation increases its stability and half-life, rendering microtubules more resistant to druginduced depolymerization and disassembly (Matsuyama et al., 2002; Szyk et al., 2014). Previous studies showed that METH impacts actin filaments, leading to actin depolymerization, altered cell shape and local accumulation of condensed actin, which may compromise TJs function and BBB integrity (Park et al., 2013; Young et al., 2014). Using the bEnd. 3 endothelial cell model, we have previously shown that METH-exposure led to a dramatic loss of F-actin integrity concomitant with claudin- 5 downregulation and redistribution (Fernandes et al., 2014). In endothelial cells, these changes at the cytoskeleton level may lead to loss of adhesion and/or relevant restructuring of the endothelium, thus contributing to METH neurotoxicity. Here, in addition, we show that METH reduces microtubule acetylation in endothelial cells. This effect is expected to contribute to BBB disruption, since it was previously shown that induced deacetylation of microtubules was associated with increased vascular permeability (Bogatcheva et al., 2007), while augmenting microtubule acetylation seems to be concomitant with the 
inhibition of barrier-disruptive pathways (Gorshkov et al., 2012; Saito et al., 2011). HDACs modulation by psychostimulants was previously reported. Exposure of adult rats to METH led to altered expression patterns of several HDACs, including HDAC1 and HDAC2 (Jayanthi et al., 2014; Martin et al., 2012), and HDAC6, HDAC8-HDAC11, SIRT2, SIRT5 and SIRT6 (Omonijo et al., 2014). These expression patterns seem to depend on the METH dose and period of exposure. Similarly, cocaine self-administration was shown to induce increased expression of several HDACs (Host et al., 2011). In the present study, to verify if METH-induced deacetylation of a-tubulin was a result of HDACs modulation by METH, we used the HDACs inhibitor TSA (Dompierre et al., 2007). Our results evidence that TSA not only prevents METH-induced deacetylation, as it strongly promotes a-tubulin acetylation also in METH-exposed endothelial cells. TSAmediated hyperacetylation was shown to increase the lifespan of neuronal cultures, and a number of studies reported the anti-inflammatory and neuroprotective effects of hyperacetylation both in vitro or in vivo models of ischemic stroke (Chuang et al., 2009; Harrison and Dexter, 2013) and toxicity (Agudelo et al., 2011). TSA was also used as a neuroprotective agent in several models of Parkinsonism (Harrison and Dexter, 2013), Huntington's disease, Alzheimer's disease and other neurological diseases (Chuang et al., 2009). In the oncology context, TSA has been intensively studied. Of note, a recent study showed that TSA inhibits cancer invasion through MMPs inhibition associated with HDAC2 suppression (Wang et al., 2013). As we have recently shown that METH disrupts actin integrity through MMP-9 modulation, it is possible that MMPs inhibition may also contribute to prevent METH-induced deacetylation of tubulin. However, as prolonged treatment with TSA may lead to neuronal cell toxicity, care should be taken when TSA is used for neuroprotective purposes (Chuang et al., 2009). Since we have recently shown that ALC counteracts the effect of METH in MMP-mediated toxicity, we have also addressed the possible role of ALC in preventing METH-modulation of HDACs. As a result, in the present work, we show that $1 \mathrm{mM} \mathrm{ALC}$ was able to prevent METH-induced deacetylation of a-tubulin. A previous report postulated that ALC had the potential to directly inhibit HDACs activity in several cell types possibly by chelating and removing $\mathrm{Zn} 2+$ from the active site of the HDAC enzyme (Huang et al., 2012). However, here, we could not observe a significant effect of ALC on HDACs activity. Of note, the doses used by Huang et al. (2012) were 5 and 10 fold higher than the $1 \mathrm{mM}$ dose shown here to efficiently prevent tubulin deacetylation. In addition, neither METH nor ALC led to altered levels of HDAC6 gene expression, which seems to indicate that both ALC- and METH-modulation of HDAC6 activity may occur in a post-translation way, as described for TSA (Xu et al., 2007). Despite the previously reported protective properties of ALC (Pettegrew et al., 2000), its action at the cytoskeleton level is still poorly explored. We have recently shown that ALC effectively prevents METH-induced loss of alignment in endothelial actin filaments, through ILK mediated release of MMP-9 (Fernandes et al., 2014). Of note, ILK was also shown to mediate MMP-9- release through regulation of glycogen synthase (GSK)-3b activity (for review see McDonald et al., 2008), while GSK$3 \mathrm{~b}$ is known to interact and colocalize with HDAC6, enhancing its activity (Li et al., 2013). Since it was reported that ALC antagonizes GSK-3b, arresting microtubule-associated protein tau hyperphosphorylation in Alzheimer's disease (Jiang et al., 2011), it is possible that ALC mediation of HDAC6 activity may also occur through GSK-3b inhibition. This putative mechanism of action is essentially different from that of TSA that may inhibit HDCAs by binding to zinc in their catalytic site (Finnin et al., 1999). In addition to hyperacetylation of the respective substrates, HDACs inhibition is also known to lead to 
increased histone acetylation and consequent chromatin remodeling (Li et al., 2013; Xu et al., 2007). Under TSA treatment, chromatin relaxation is known to increase the expression of CDKN1A (cyclin-dependent kinases inhibitor, or p21) gene (Blagosklonny et al., 2002; Hrabeta et al., 2014; Ocker and Schneider-Stock, 2007; Xu et al., 2007). As such, CDKN1A (p21) expression is frequently used as a marker of HDAC inhibition (Harrison and Dexter, 2013; Huang et al., 2012; Xu et al., 2007). As expected our results show that TSA promotes the overexpression of CDKN1A. Opposing to this, in METHexposed cells, CDKN1A expression was clearly reduced, which was counteracted by ALC and TSA. These results show that in the presence of METH, both ALC and TSA seem to maintain CDKN1A expression at levels that are not significantly different from the control. However, contrarily to TSA, ALC does not trigger CDKN1A overexpression, which has been repeatedly associated with cell cycle arrest, senescence and cell death (reviewed by Xu et al., 2007). In that sense, the protection afforded through ALC may be more effective and lead to less severe side effects.
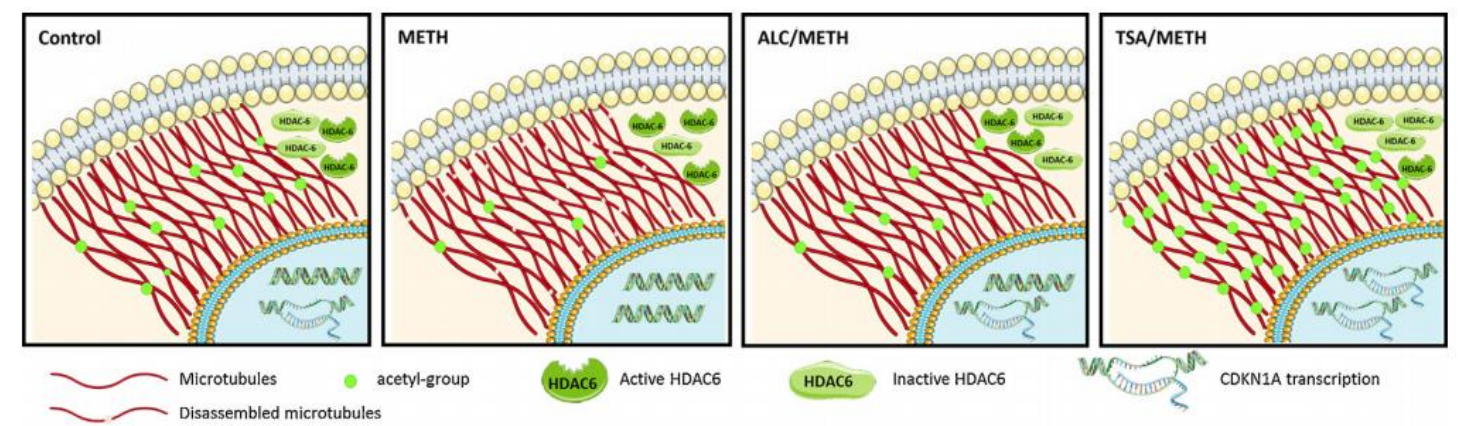

Fig. 5. Schematic representation of METH-induced deacetylation of microtubules, which is-prevented by ALC. METH induces $\alpha$-tubulin deacetylation through modulation of HDAC6 activity. Microtubules deacetylation is represented by disassembly patterns previously described (Matsuyama et al., 2002; Perdiz et al., 2011). ALC pretreatment prevents METH-induced deacetylation, resulting in higher levels of $\alpha$-tubulin acetylation (ALC/METH). Compared to the strong HDACs inhibition obtained with TSA, the protective action provided by ALC preserves microtubule acetylation without promoting hyperacetylation and increased transcription of CDKN1A (p21) (TSA/METH).

In summary, we show here for the first time that METH promotes deacetylation of atubulin, which is likely to impact the structure of microtubules at the endothelial cytoskeleton and disturb the BBB function. Additionally, we show also that ALC is able to prevent METHinduced deacetylation, providing effective protection of microtubules acetylation, without risking the critical consequences of HDACs inhibition (Fig. 5). Although further investigation is still needed, HDACs regulation may become a new therapeutic target for ALC.

\section{References}

Agudelo, M., Gandhi, N., Saiyed, Z., Pichili, V., Thangavel, S., Khatavkar, P., YndartArias, A., Nair, M., 2011. Effects of alcohol on histone deacetylase 2 (HDAC2) and the neuroprotective role of trichostatin A (TSA). Alcohol. Clin. Exp. Res. 35, 1550-1556. doi:http://dx.doi.org/10.1111/j.1530-0277.2011.01492.x.

Alves, E., Binienda, Z., Carvalho, F., Alves, C.J., Fernandes, E., de Lourdes Bastos, M., Tavares, M.A., Summavielle, T., 2009. Acetyl-L-carnitine provides effective in vivo neuroprotection over 3,4-methylenedioximethamphetamine-induced mitochondrial neurotoxicity in the adolescent rat brain. Neuroscience 158, 514-523. doi:http://dx.doi.org/10.1016/j.neuroscience.2008.10.041. 
Blagosklonny, M.V., Robey, R., Sackett, D.L., Du, L., Traganos, F., Darzynkiewicz, Z., Fojo, T., Bates, S.E., 2002. Histone deacetylase inhibitors all induce p21 but differentially cause tubulin acetylation, mitotic arrest, and cytotoxicity. Mol. Cancer Ther. 1, 937-941.

Bogatcheva, N.V., Adyshev, D., Mambetsariev, B., Moldobaeva, N., Verin, A.D., 2007. Involvement of microtubules, p38, and rho kinases pathway in 2-methoxyestradiol-induced lung vascular barrier dysfunction. Am. J. Physiol. 292, L487-L499. doi:http://dx.doi.org/10.1152/ajplung.00217.2006.

Cassel, S., Carouge, D., Gensburger, C., Anglard, P., Burgun, C., Dietrich, J.B., Aunis, D., Zwiller, J., 2006. Fluoxetine and cocaine induce the epigenetic factors MeCP2 and MBD1 in adult rat brain. Mol. Pharmacol. 70, 487-492. doi:http://dx.doi. org/10.1124/mol.106.022301.

Chuang, D.M., Leng, Y., Marinova, Z., Kim, H.J., Chiu, C.T., 2009. Multiple roles of HDAC inhibition in neurodegenerative conditions. Trends Neurosci. 32, 591-601. doi:http://dx.doi.org/10.1016/j.tins.2009.06.002.

Conant, K., Lonskaya, I., Szklarczyk, A., Krall, C., Steiner, J., Maguire-Zeiss, K., Lim, S.T., 2011. Methamphetamine-associated cleavage of the synaptic adhesion molecule intercellular adhesion molecule-5. J. Neurochem. 118, 521-532. doi: http://dx.doi.org/10.1111/j.14714159.2010.07153.x.

Dietrich, J.B., 2009. Alteration of blood-brain barrier function by methamphetamine and cocaine. Cell Tissue Res. 336, 385-392. doi:http://dx.doi.org/10.1007/ s00441-009-0777-y.

Dompierre, J.P., Godin, J.D., Charrin, B.C., Cordelieres, F.P., King, S.J., Humbert, S., Saudou, F., 2007. Histone deacetylase 6 inhibition compensates for the transport deficit in Huntington's disease by increasing tubulin acetylation. J. Neurosci. 27, 3571-3583. doi:http://dx.doi.org/10.1523/JNEUROSCI.0037-07.2007.

Fernandes, S., Salta, S., Bravo, J., Silva, A.P., Summavielle, T., 2014. Acetyl-L-carnitine prevents methamphetamine-induced structural damage on endothelial cells via ILK-related MMP-9 activity. Mol. Neurobiol. 1-15. doi:http://dx.doi.org/ 10.1007/s12035-014-8973-5.

Finnin, M.S., Donigian, J.R., Cohen, A., Richon, V.M., Rifkind, R.A., Marks, P.A., Breslow, R., Pavletich, N.P., 1999. Structures of a histone deacetylase homologue bound to the TSA and SAHA inhibitors. Nature 401, 188-193. doi:http://dx.doi. org/10.1038/43710.

Gold, M.S., Kobeissy, F.H., Wang, K.K., Merlo, L.J., Bruijnzeel, A.W., Krasnova, I.N., Cadet, J.L., 2009. Methamphetamine- and trauma-induced brain injuries: comparative cellular and molecular neurobiological substrates. Biol. Psychiat. 66, 118-127. doi:http://dx.doi.org/10.1016/j.biopsych.2009.02.021.

Gold, W.A., Lacina, T.A., Cantrill, L.C., Christodoulou, J., 2015. MeCP2 deficiency is associated with reduced levels of tubulin acetylation and can be restored using HDAC6 inhibitors. J. Mol. Med. 93, 63-72. doi:http://dx.doi.org/10.1007/s00109- 014-1202-x.

Goncalves, J., Baptista, S., Martins, T., Milhazes, N., Borges, F., Ribeiro, C.F., Malva, J.O., Silva, A.P., 2010. Methamphetamine-induced neuroinflammation and neuronal dysfunction in the mice hippocampus: preventive effect of indomethacin. Eur. J. Neurosci. 31, 315-326. doi:http://dx.doi.org/10.1111/j.1460-9568.2009.07059.x. 
Gorshkov, B.A., Zemskova, M.A., Verin, A.D., Bogatcheva, N.V., 2012. Taxol alleviates 2methoxyestradiol-induced endothelial permeability. Vasc. Pharmacol. 56, 56-63. doi:http://dx.doi.org/10.1016/j.vph.2011.10.002.

Hammond, J.W., Cai, D.W., Verhey, K.J., 2008. Tubulin modifications and their cellular functions. Curr. Opin. Cell Biol. 20, 71-76. doi:http://dx.doi.org/10.1016/ j.ceb.2007.11.010.

Haorah, J., Floreani, N.A., Knipe, B., Persidsky, Y., 2011. Stabilization of superoxide dismutase by acetyl-L-carnitine in human brain endothelium during alcohol exposure: novel protective approach. Free Rad. Biol. Med. 51, 1601-1609. doi: http://dx.doi.org/10.1016/i.freeradbiomed.2011.06.020.

Harrison, I.F., Dexter, D.T., 2013. Epigenetic targeting of histone deacetylase: therapeutic potential in Parkinson's disease? Pharmacol. Ther. 140, 34-52. doi: http://dx.doi.org/10.1016/j.pharmthera.2013.05.010.

Host, L., Dietrich, J.-B., Carouge, D., Aunis, D., Zwiller, J., 2011. Cocaine selfadministration alters the expression of chromatin-remodelling proteins; modulation by histone deacetylase inhibition. J. Psychopharmacol. (Oxford. England) 25, 222-229. doi:http://dx.doi.org/10.1177/0269881109348173.

Hrabeta, J., Stiborova, M., Adam, V., Kizek, R., Eckschlager, T., 2014. Histone deacetylase inhibitors in cancer therapy. A review. Biomed. Pap. Med. Fac. Univ. Palacky, Olomouc, Czechoslovakia 158, 161-169. doi:http://dx.doi.org/10.5507/ bp.2013.085.

Huang, H., Liu N, Guo H., Liao, S., Li, X., Yang, C., Song, Liu S., W, Liu C., Guan, L., Li, B., Xu, L., Zhang, C., Wang, X., Dou Q.P, Liu J., 2012. L-Carnitine is an endogenous HDAC inhibitor selectively inhibiting cancer cell growth in vivo and in vitro. PloS One 7, e49062. doi:http://dx.doi.org/10.1371/journal.pone.0049062.

Hubbert, C., Guardiola, A., Shao, R., Kawaguchi, Y., Ito, A., Nixon, A., Yoshida, M., Wang, X.-F., Yao, T.-P., 2002. HDAC6 is a microtubule-associated deacetylase. Nature 417, 455-458.

Jayanthi, S., McCoy, M.T., Chen, B., Britt, J.P., Kourrich, S., Yau, H.J., Ladenheim, B., Krasnova, I.N., Bonci, A., Cadet, J.L., 2014. Methamphetamine downregulates striatal glutamate receptors via diverse epigenetic mechanisms. Biol. Psych. 76, 47-56. doi:http://dx.doi.org/10.1016/j.biopsych.2013.09.034.

Jiang, X., Tian, Q., Wang, Y., Zhou, X.W., Wang, Xie J.Z., J.Z, Zhu L.Q., 2011. Acetyl-Lcarnitine ameliorates spatial memory deficits induced by inhibition of phosphoinositol-3 kinase and protein kinase C. J. Neurochem.118, 864-878. doi: http://dx.doi.org/10.1111/j.14714159.2011.07355.x.

Jin, C.H., Moo, T.T., Youn, C.S., Sun, Y.J., Joo-II, K., Sook, H.E., Onyou, H., 2002. Methamphetamine-induced apoptosis in a CNS-derived catecholaminergic cell Line. Mol. Cells 13, 221-227.

Kousik, S.M., Napier, T.C., Carvey, P.M., 2012. The effects of psychostimulant drugs on blood brain barrier function and neuroinflammation. Front. Pharmacol. 3, 121. doi:http://dx.doi.org/10.3389/fphar.2012.00121.

Krasnova, I.N., Cadet, J.L., 2009. Methamphetamine toxicity and messengers of death. Brain Res. Rev. 60, 379-407. doi:http://dx.doi.org/10.1016/j. brainresrev.2009.03.002. Li, Y., Shin, D., 
Kwon, S.H., 2013. Histone deacetylase 6 plays a role as a distinct regulator of diverse cellular processes. FEBS J. 280, 775-793. doi:http://dx.doi. org/10.1111/febs.12079.

Martin, T.A., Jayanthi, S., McCoy, M.T., Brannock, C., Ladenheim, B., Garrett, T., Lehrmann, E., Becker, K.G., Cadet, J.L., 2012. Methamphetamine causes differential alterations in gene expression and patterns of histone acetylation/ hypoacetylation in the rat nucleus accumbens. PloS One 7, e34236. doi:http:// dx.doi.org/10.1371/journal.pone.0034236.

Martins, T., Baptista, S., Goncalves, J., Leal, E., Milhazes, N., Borges, F., Ribeiro, C.F., Quintela, O., Lendoiro, E., Lopez-Rivadulla, M., Ambrosio, A.F., Silva, A.P., 2011. Methamphetamine transiently increases the blood-brain barrier permeability in the hippocampus: role of tight junction proteins and matrix metalloproteinase-9. Brain Res. 1411, 28-40. doi:http://dx.doi.org/10.1016/j. brainres.2011.07.013.

Martins, T., Burgoyne, T., Kenny, B.A., Hudson, N., Futter, C.E., Ambrosio, A.F., Silva, A. P., Greenwood, J., Turowski, P., 2013. Methamphetamine-induced nitric oxide promotes vesicular transport in blood-brain barrier endothelial cells. Neuropharmacology 65, 74-82. doi:http://dx.doi.org/10.1016/j. neuropharm.2012.08.021.

Matsuyama, A., Shimazu, T., Sumida, Y., Saito, A., Yoshimatsu, Y., Seigneurin-Berny, D., Osada, H., Komatsu, Y., Nishino, N., Khochbin, S., Horinouchi, S., Yoshida, M., 2002. In vivo destabilization of dynamic microtubules by HDAC6-mediated deacetylation. EMBO J. 21, 68206831.

McDonald, P.C., Fielding, A.B., Dedhar, S., 2008. Integrin-linked kinase?essential roles in physiology and cancer biology. J. Cell Sci. 121, 3121-3132. doi:http://dx. doi.org/10.1242/jcs.017996.

Muneer, P.M.A., Alikunju, S., Szlachetka, A.M., Haorah, J., 2011. Methamphetamine inhibits the glucose uptake by human neurons and astrocytes: stabilization by acetyl-L-carnitine. PloS One 6, e19258. doi:http://dx.doi.org/10.1371/journal. pone.0019258.

Nahhas, F., Dryden, S.C., Abrams, J., Tainsky, M.A., 2007. Mutations in SIRT2 deacetylase which regulate enzymatic activity but not its interaction with HDAC6 and tubulin. Mol. Cell. Biochem. 303, 221-230. doi:http://dx.doi.org/ 10.1007/s11010-007-9478-6.

Northrop, N.A., Yamamoto, B.K., 2012. Persistent neuroinflammatory effects of serial exposure to stress and methamphetamine on the blood-brain barrier. J. Neuro. Pharmacol. 7, 951-968. doi:http://dx.doi.org/10.1007/s11481-012-9391-y.

Ocker, M., Schneider-Stock, R., 2007. Histone deacetylase inhibitors: signalling towards p21cip1/waf1. Int. J. Biochem. Cell Biol. 39, 1367-1374. doi:http://dx. doi.org/10.1016/j.biocel.2007.03.001.

Omonijo, O., Wongprayoon, P., Ladenheim, B., McCoy, M.T., Govitrapong, P., Jayanthi, S., Cadet, J.L., 2014. Differential effects of binge methamphetamine injections on the mRNA expression of histone deacetylases (HDACs) in the rat striatum. Neurotoxicology 45, 178-184. doi:http://dx.doi.org/10.1016/j. neuro.2014.10.008.

Park, M., Kim, H.J., Lim, B., Wylegala, A., Toborek, M., 2013. Methamphetamineinduced occludin endocytosis is mediated by the Arp2/3 complex-regulated actin rearrangement. J. Biol. Chem. 288 (33), 33324-33334. doi:http://dx.doi. org/10.1074/jbc.M113.483487. 
Perdiz, D., Mackeh, R., Pous, C., Baillet, A., 2011. The ins and outs of tubulin acetylation: more than just a post-translational modification? Cell. Signal. 23, 763-771. doi:http://dx.doi.org/10.1016/j.cellsig.2010.10.014.

Pettegrew, J., Levine, J., McClure, R., 2000. Acetyl-L-carnitine physical-chemical, metabolica, and therapeutic properties: relevance for its mode of action in Alzheimer's disease and geriatric depression. Mol. Psychiat. 5, 616-632.

Ramirez, S.H., Potula, R., Fan, S., Eidem, T., Papugani, A., Reichenbach, N., Dykstra, H., Weksler, B.B., Romero, I.A., Couraud, P.O., Persidsky, Y., 2009. Methamphetamine disrupts blood-brain barrier function by induction of oxidative stress in brain endothelial cells. J. Cereb. Blood Flow Metab. 29, 1933-1945. doi:http://dx.doi.org/10.1038/jcbfm.2009.112.

Sadoul, K., Wang, J., Diagouraga, B., Khochbin, S., 2011. 2011. The tale of protein lysine acetylation in the cytoplasm. J. Biomed. Biotechnol. 970, 382. doi:http:// dx.doi.org/10.1155/2011/970382.

Saito, S., Lasky, J.A., Nguyen, H., Danchuk, S., Sullivan, D.E., Shan, B., 2011. Pharmacological inhibition of HDAC6 attenuates endothelial barrier dysfunction induced by thrombin. Biochem. Biophys. Res. Commun. 408, 630-634. doi:http://dx.doi.org/10.1016/j.bbrc.2011.04.075.

Song, Y., Brady, S.T., 2014. Post-translational modifications of tubulin: pathways to functional diversity of microtubules. Trends Cell Biol. doi:http://dx.doi.org/ 10.1016/j.tcb.2014.10.004.

Szyk, A., Deaconescu, A.M., Spector, J., Goodman, B., Valenstein, M.L., Ziolkowska, N. E., Kormendi, V., Grigorieff, N., Roll-Mecak, A., 2014. Molecular basis for agedependent microtubule acetylation by tubulin acetyltransferase. Cell 157, 1405-1415. doi:http://dx.doi.org/10.1016/j.cell.2014.03.061.

Tu, C.Y., Chen, C.H., Hsia, T.C., Hsu, H., Wei, L., Yu, M.C., Chen, W.S., Hsu, W., Yeh, H., Liu, C., Chen, Y.J., Huang, W.C., 2014. Trichostatin A suppresses EGFR expression through induction of micro RNA-7 in an HDAC-independent manner in lapatinib-treated cells. Biomed. Res. Int. 2014 doi:http://dx.doi.org/10.1155/ 2014/168949.

Urrutia, A., Rubio-Araiz, A., Gutierrez-Lopez, M.D., ElAli, A., Hermann, D.M., O'Shea, E., Colado, M.I., 2013. A study on the effect of JNK inhibitor, SP600125, on the disruption of blood-brain barrier induced by methamphetamine. Neurobiol. Dis. 50, 49 - 58. doi:http://dx.doi.org/10.1016/j.nbd.2012.10.006.

Wang, F., Qi, Y., Li, X., He, W., Fan, Q.X., Zong, H., 2013. HDAC inhibitor trichostatin A suppresses esophageal squamous cell carcinoma metastasis through HADC2 reduced MMP-2/9. Clin. Invest. Med. 36, E87 - E94.

Xu, W.S., Parmigiani, R.B., Marks, P.A., 2007. Histone deacetylase inhibitors: molecular mechanisms of action. Oncogene 26, 5541-5552. doi:http://dx.doi. org/10.1038/sj.onc.1210620.

Yang, X.J., Seto, E., 2008. Lysine acetylation: codified crosstalk with other posttranslational modifications. Mol. Cell 31, 449-461. doi:http://dx.doi.org/ 10.1016/j.molcel.2008.07.002.

Young, E.J., Aceti, M., Griggs, E.M., Fuchs, R.A., Zigmond, Z., Rumbaugh, G., Miller, C. A., 2014. Selective, retrieval-independent disruption of methamphetamineassociated memory by actin 
depolymerization. Biol. Psychiat.

http://dx.doi.org/10.1016/j.biopsych.2013.07.036.

75 ,

96-104.

doi: 